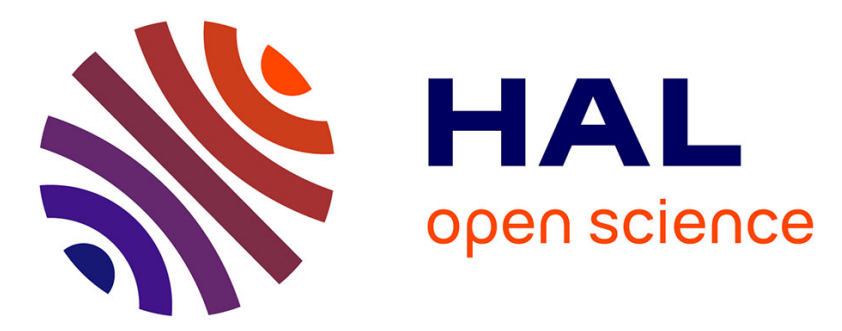

\title{
The magnetic field diffusion equation including dynamic, hysteresis: A linear formulation of the problem
}

\author{
Marie-Ange Raulet, Benjamin Ducharne, Jean-Pierre Masson, G. Bayada
}

\section{To cite this version:}

Marie-Ange Raulet, Benjamin Ducharne, Jean-Pierre Masson, G. Bayada. The magnetic field diffusion equation including dynamic, hysteresis: A linear formulation of the problem. IEEE Transactions on Magnetics, 2004, 40 (2 Part 2), pp.872-875. 10.1109/TMAG.2004.824816 . hal-00141389

\section{HAL Id: hal-00141389 \\ https://hal.science/hal-00141389}

Submitted on 18 Apr 2007

HAL is a multi-disciplinary open access archive for the deposit and dissemination of scientific research documents, whether they are published or not. The documents may come from teaching and research institutions in France or abroad, or from public or private research centers.
L'archive ouverte pluridisciplinaire HAL, est destinée au dépôt et à la diffusion de documents scientifiques de niveau recherche, publiés ou non, émanant des établissements d'enseignement et de recherche français ou étrangers, des laboratoires publics ou privés. 


\title{
The Magnetic Field Diffusion Equation Including Dynamic Hysteresis: A Linear Formulation of the Problem
}

\author{
M. A. Raulet, B. Ducharne, J. P. Masson, and G. Bayada
}

\begin{abstract}
The introduction of accurate material modeling such as hysteresis phenomenon in numerical field calculation leads to numerical problems induced by the nonlinear properties of the initial system. We focus on the solution of the magnetic field diffusion equation, which contains such problems. This paper presents a new formulation of the diffusion equation including dynamic hysteresis. The resulting formulation leads to a linear system to solve. A numerical implementation of the problem and an experimental validation are also presented.
\end{abstract}

Index Terms-Diffusion equations, dynamics, hysteresis, linear systems.

\section{INTRODUCTION}

$\mathbf{S}$ EVERAL works are effected on the coupling of accurate material laws with Maxwell's equations. The integration of a hysteretic model in a magnetostatic or magnetodynamic problem needs the use of particular techniques such as the fixedpoint or the Newton-Raphson methods [1]-[3].

In our team, we focus on the magnetic field diffusion in the cross section of a magnetic lamination.

We already implemented a hysteretic dynamic model in the diffusion equation of the magnetic excitation field [4]. The resolution of the whole diffusion model leads to accurate results, but numerical problems of convergence appear in particular cases.

In this paper, we present how a new formulation of the diffusion problem coupled to a hysteretic dynamic material law leads to a linear system to be solved.

A numerical formulation of this problem is detailed in this paper. The implementation must be modular, flexible, and must necessitate low memory allocation and short calculation time.

Several simulation results and an experimental validation of this new diffusion model are presented.

\section{PROBLEM}

\section{A. Hypothesis}

The diffusion equation is solved through a cross section sheet of an Epstein frame. The design assumptions are those of the

Manuscript received July 1, 2003.

M. A. Raulet, B. Ducharne, and J. P. Masson are with CEGELY, C.N.R.S. UPRESA 5005, Université Claude Bernard, 69622 Villeurbanne Cedex, France (raulet@cegely.univ-lyon.fr).

G. Bayada is with MAPLY, C.N.R.S. UMR 5585, INSA de Lyon, 69621

Villeurbanne Cedex, France.

Digital Object Identifier 10.1109/TMAG.2004.824816
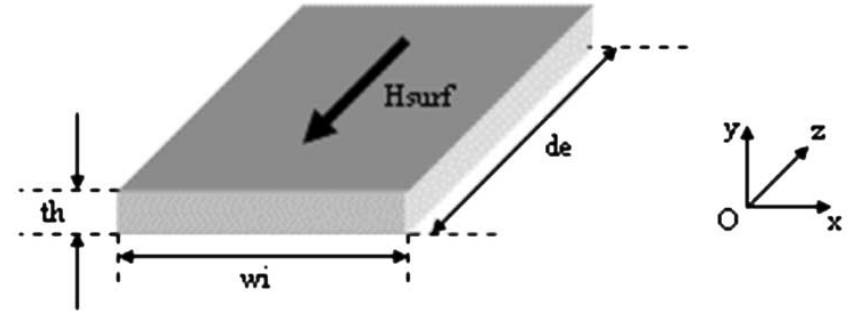

Fig. 1. Lamination on study.

Epstein frame (Fig. 1): unidirectional and homogeneous surface excitation field (according to the depth of the sheet). In addition, we can consider the conductivity $\sigma$ of the magnetic sheet as constant and homogeneous.

Taking into account the dimensions of the studied sheets [width (wi) much more significant than the thickness (th)], a one-dimensional (1-D) study according to the thickness of the sheet is carried out [5]. Symmetry considerations lead to limit the studied area to the half thickness (th/2) of the sheet. This 1-D study is especially effected in order to limit the number of the variables of the problem. This simplification constitutes a significant profit with the integration of a hysteresis model in a field computation.

\section{B. Dynamic Material Law}

If a static reversible material law $B(H)$ is considered in the diffusion equation, the resolution is easy but leads to inaccurate results. In this case, the dynamic effects related to the wall motions and the hysteretic phenomenon are neglected.

In a previous work, we have shown that, considering in the same manner the eddy-current effects and the wall motion effects, it is possible to describe the dynamical behavior of a magnetic flux tube (with constant cross section) using a state representation with a static feedback [6]. This feedback represents the static characteristic of the circuit. This model needs only one parameter, which is in low dependence of the magnetic excitation conditions (waveforms, frequency, amplitude).

A lot of experiments have shown that the accuracy of this representation is increased when wall motion effects are preponderant.

As the distribution of magnetic domains is statistically distributed in all directions of polycrystalline material, we propose to use the same representation to describe the dynamic effects 
due to the wall motions. In the purpose, we use the differential equation

$$
\frac{\mathrm{dB}}{\mathrm{dt}}=\frac{1}{\beta}\left[H-H_{\text {stat }}(B)\right]
$$

where $B$ represents the local magnetic field, and $H$ is the local magnetic resulting excitation field.

$H_{\text {stat }}(B)$ is a fictitious value calculated from a static model of material law. A static hysteretic model is integrated in (1).

Several static hysteretic models exist. For the first time, we have chosen Preisach-Néel's model.

The parameter $\beta$ is identified with only one dynamic $B(H)$ characteristic performed in any experimental conditions.

Specific works have shown that this $\beta$ parameter can be considered as independent of the geometry and of the studied sample and of its excitation. A lot of experimental validations have shown that this dynamic material law constitutes a good description of dynamic effects due to Bloch's wall motions [7].

This material law represents a statistical behavior of the wall motions. It can then be considered as isotropic and characteristic of the material.

A coupled resolution of dynamic material law and the magnetic field diffusion equation must be effected. The magnetic diffusion equation (2) results from Maxwell's equations and a law, which describes the conductive property of the material

$$
\overrightarrow{\operatorname{rot}}(\overrightarrow{\operatorname{rot}} \vec{H})=-\sigma \frac{\partial \vec{B}}{\partial t}
$$

As the magnetic field is always perpendicular to the cross section, $\operatorname{div}(H)=0$, so (2) becomes

$$
\frac{\partial^{2} H(y, t)}{\partial y^{2}}=\sigma \cdot \frac{\partial B(y, t)}{\partial t} .
$$

In a previous work, the temporal material law (1), independent of excitation conditions, has been integrated in (3) using the permeability $\mu=B / H$. The local permeability $\mu(y, t)$ was calculated from the resolution of (1).

A discretization of the second term of (3) led to

$$
\begin{aligned}
\frac{\partial^{2} H(y, t)}{\partial y^{2}}=\frac{\sigma}{d t} \cdot(\mu(y, t) & \cdot H(y, t) \\
& -\mu(y, t-d t) \cdot H(y, t-d t)) .
\end{aligned}
$$

The finite-element formulation of the problem leads to obtain a nonlinear system.

The Newton-Raphson's algorithm has solved this system. The resolution gives accurate results but sometimes presents numerical problems of convergence related to the nonlinearity [4].

\section{NeW Formulation OF THE DifFusion PROBLEM}

\section{A. Principle}

The idea of the new formulation comes from (1), which defines the law of the material dynamic property. The $\mathrm{dB} / \mathrm{dt}$ term of (1) constitutes also a part of the second term of the diffusion equation (3).

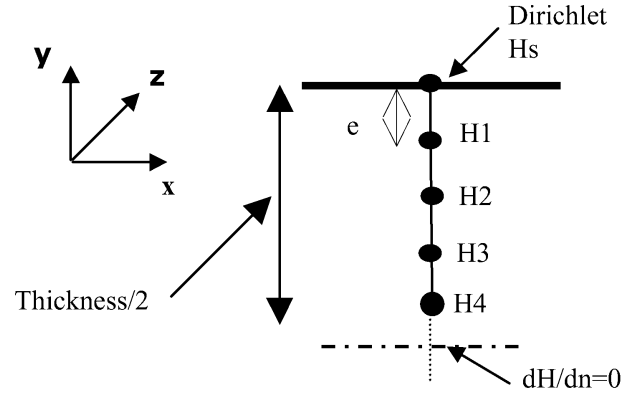

Fig. 2. One-dimensional problem.

It is natural to replace the $\mathrm{dB} / \mathrm{dt}$ term of (3) by the second member of (1), which gives a new formulation of the diffusion equation

$$
\frac{\partial^{2} H(y, t)}{\partial y^{2}}=\sigma \cdot \frac{1}{\beta} \cdot\left[H(y, t)-H_{\text {stat }}(B(y, t))\right] .
$$

This equation coupled with (1), which defines the dynamic material law, does not reveal any more the term of permeability $\mu$, which is responsible of the nonlinearity of the system to be solved.

\section{B. Reduction of the Nonlinearity Effect}

Taking into account the simplicity of the study domain (half thickness th $/ 2$ of the sheet illustrated on Fig. 2), a formulation by finite difference method of the diffusion equation is carried out.

The new system obtained is

$$
[M] \cdot[H]=\left[S_{1}\right]+\left[S_{2}\right]
$$

where

$$
[M]=\left[\begin{array}{cccc}
\frac{\sigma e^{2}}{\beta}+2 & -1 & 0 & 0 \\
-1 & \frac{\sigma e^{2}}{\beta}+2 & -1 & 0 \\
0 & -1 & \frac{\sigma e^{2}}{\beta}+2 & -1 \\
0 & 0 & -2 & \frac{\sigma e^{2}}{\beta}+2
\end{array}\right]
$$

is the stiffness matrix. It contains only constant terms, so its inversion is performed only once.

$[H]$ is the vector of the unknown excitation fields at each node of the mesh

$$
\left[S_{1}\right]=\left[\begin{array}{c}
H_{\text {surf }} \\
0 \\
0 \\
0
\end{array}\right] \quad\left[S_{2}\right]=\left[\begin{array}{c}
\frac{\sigma e^{2}}{\beta} \cdot H 1_{\text {stat }}\left(B_{1}\right) \\
\frac{\sigma e^{2}}{\beta} \cdot H 2_{\text {stat }}\left(B_{2}\right) \\
\frac{\sigma e^{2}}{\beta} \cdot H 3_{\text {stat }}\left(B_{3}\right) \\
\frac{\sigma e^{2}}{\beta} \cdot H 4_{\text {stat }}\left(B_{4}\right)
\end{array}\right]
$$

[ $\left.S_{1}\right]$ only depends on the excitation field $H_{\text {surf }}$ on the surface of the sheet and on the initial conditions.

$\left[S_{2}\right]$ is a term made up of values $H_{\text {stat }}(B i)$, which come from a static hysteretic model. The nonlinearity of the problem is included in this term.

The solution of the system can be reached without iteration when implicit Euler's method is chosen for $\left[S_{2}\right]$ calculation. So 


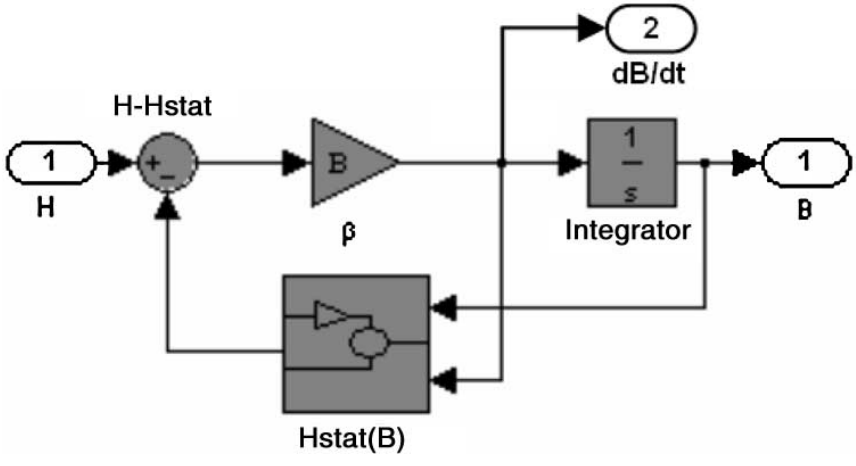

Fig. 3. Dynamic material law block diagram.

convergence problems are eliminated and the computation time is reduced.

\section{NUMERICAL IMPLEMENTATION}

The new formulation of the full diffusion problem implies to solve a linear system which couples partial derivative equations and differential equations. We detail now, how an implementation of the whole diffusion model can be effected.

We chose Matlab for several reasons: first, its broad distribution throughout the world; second, because it is convivial and many preprogrammed blocks already exist.

The complete problem programming is carried out in several stages. Each stage is concluded by the development of subsystems materialized by blocks known as "macro-block;" the assembly of these macro-blocks allows the development of the final model.

\section{A. Programming of the Differential Equation "Dynamic Material Law"}

The installation of the differential equation (1) known as "dynamic material law" requires the use of a static hysteresis model $H_{\text {stat }}(B)$.

This static model is compacted in a single Simulink block in order to facilitate its integration in the dynamic material law model and to not overload the diagram final block.

Fig. 3 presents the resolution Simulink block diagram for the dynamical material law.

\section{B. Programming of the Complete Model}

System (6) is linear and the stiffness matrix is positive definite. This condition is sufficient to allow us to use a Cholesky resolution method directly available in the Matlab library.

The output of the block "Cholesky resolution" generates a vector corresponding to the magnetic excitation fields of each node of the mesh. These excitation fields are used then as excitation for the various blocks "dynamic material law."

From these blocks "dynamic material law," we have local induction corresponding to each node of the grid. Finally, a calculation function allows to determinate the averaged induction through the section.

Moreover, if we consider the surface field $H_{\text {surf }}$ as the magnetic excitation, it then becomes possible to return the material hysteresis loop.

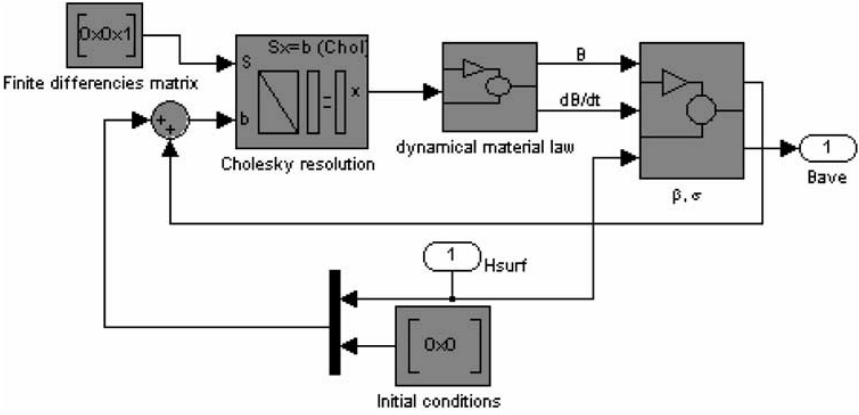

Fig. 4. Modeling of the whole system.

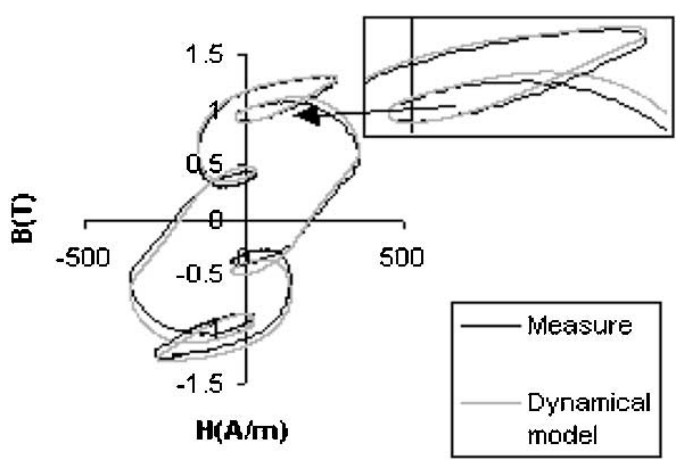

Fig. 5. Dynamic loops with $50 \mathrm{~Hz}$ sine wave excitation with harmonic 3.

Fig. 4 presents the complete model before its integration in a single block, facilitating its implementation in the whole system.

\section{EXPERIMENTAL VALIDATION}

In order to test the results of simulations corresponding to the new formulation of the whole diffusion equation, we carried out tests on Epstein frame sheets. These sheets are low silicon iron electrical alloy referenced M700P65. The thickness is $0.65 \mathrm{~mm}$; the width is $30 \mathrm{~mm}$. The $\sigma$ conductivity is identified to the value of $4.25 \times 10^{6}(\Omega \cdot \mathrm{m})^{-1}$.

The resolution of the diffusion equation gives the local values of $B(y, t)$, which can be used to calculate the whole flux. Only this last value can be compared with experimental data.

Fig. 5 shows the superimposition of simulated and measured averaged dynamic loops when the surface excitation field $H_{\text {surf }}$ is $50 \mathrm{~Hz}$ frequency sine wave with $50 \%$ of harmonic 3 . We can note the accuracy of a temporal model.

This simulation case had been tested with the old formulation of the diffusion equation. The new resolution eliminates any convergence problem.

For this case, the field distribution is strongly inhomogeneous in the cross section of the lamination. Figs. 6 and 7 superimpose the temporal evolution and the corresponding loops for points situated on the surface and in the center of the lamination.

As the averaged results are in good correlation with experimental results, we assume that the local distribution is accurate.

Considering this assumption, we dispose of local evaluation inaccessible to the direct measurement.

The calculation time, for the simulation presented, in Figs. 5-7 needs only $30 \mathrm{~s}$ for 10 nodes of the mesh on a PC with a $800 \mathrm{MHz}$ Pentium IV processor. 


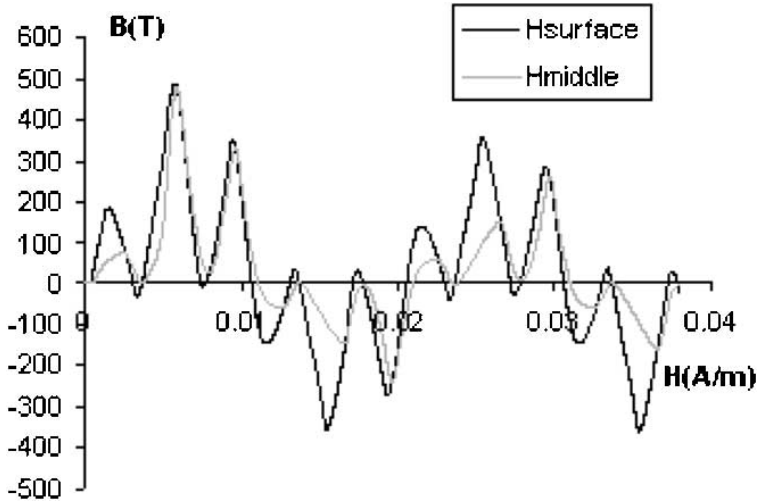

Fig. 6. Excitation field on the surface and in the center of the sheet.

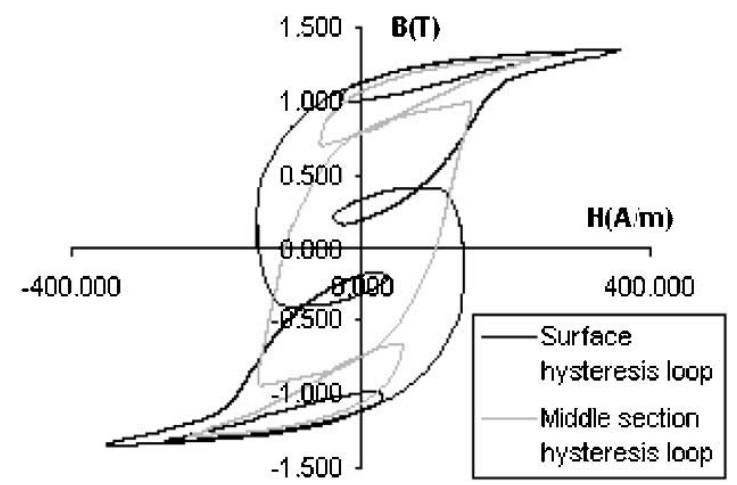

Fig. 7. Local loops on the surface and in the center of the sheet.

\section{CONCLUSION}

A new formulation of the diffusion equation including dynamic hysteresis is presented. This new formulation leads to the resolution of a linear system, which avoids many numerical problems. Local results are available. The global results are validated with experimental data. A numerical implementation of the diffusion model using flexible software is realized. The resolution is fast and modular.

The present work concerns 1-D formulation; it suits to the classical magnetic laminations. For two-dimensional uniaxial problems, a similar formulation is in progress in our team. A three-dimensional formulation is hardly justified without considering the material anisotropy. It leads to a huge problem.

\section{REFERENCES}

[1] F. Ossart and V. Ionita, "Convergence de la méthode du point fixe modifié pour le calcul de champ magnétique avec hystérésis," Eur. Phys. J. Appl. Phys., vol. AP-5, pp. 63-69, 1999.

[2] J. Saitz, "Newton-Raphson method and fixed-point technique in finite element computation of magnetic fields problems in media with hysteresis," IEEE Trans. Magn., vol. 35, pp. 1398-1401, May 1999.

[3] F. Henrotte, "Modeling ferromagnetic materials in 2D finite element problems using Preisach's model," IEEE Trans. Magn., vol. 28, pp. 2614-2616, Sept. 1992.

[4] M. A. Raulet, J. P. Masson, and D. Gaude, "Presentation and experimental validation of a behavioral model of dynamic properties for magnetic materials," J. Phys IV, vol. 8, pp. 619-622, 1998.

[5] M. A. Raulet, J. P. Masson, and A. Nicolas, "Reduction of a 2D problem of magnetic field diffusion including dynamic hysteresis through the cross section of a magnetic lamination," in COMPUMAG Conf., Sapporo, Japan, Oct. 25-28, 1999.

[6] F. Marthouret, J. P. Masson, and H. Fraisse, "Modeling of a nonlinear conductive magnetic circuit," IEEE Trans. Magn., vol. 31, pp. 4065-4070, Nov. 1995.

[7] M. A. Raulet, J. P. Masson, and H. Fraisse, "Test of the sample geometry independence of a dynamic hysteresis behavioral model of magnetic materials," J. Magn. Magn. Mater, pp. 215-216, 620-622, June 2000. 